\title{
An Implementation of Yolo-family Algorithms in Classifying the Product Quality for the ABS Metallization
}

YUH-WEN CHEN ( $\sim$ profchen@mail.dyu.edu.tw )

Da-Yeh University https://orcid.org/0000-0002-8916-8058

Jing Mau Shiu

University Rd., Dacun

\section{Research Article}

Keywords: Deep Learning, Acrylonitrile Butadiene Styrene (ABS), YOLO, Automatic Optical Inspection (AOI)

Posted Date: September 16th, 2021

DOI: https://doi.org/10.21203/rs.3.rs-898089/v1

License: (c) (i) This work is licensed under a Creative Commons Attribution 4.0 International License. Read Full License 


\title{
An Implementation of Yolo-family Algorithms in Classifying the Product Quality for the ABS Metallization
}

\author{
Yuh Wen Chen ${ }^{*}$ and Jing Mau Shiu ${ }^{2 \dagger}$ \\ $1^{*}$ Department of Information Management, Da-Yeh University, \\ No.168, University Rd., Dacun,, Changhua, 515006, Taiwan, \\ R.O.C..
}

${ }^{2}$ Department of Industrial Engineering and Management, Da-Yeh University, No.168, University Rd., Dacun,, Changhua, 515006, Taiwan, R.O.C..

*Corresponding author(s). E-mail(s): profchen@mail.dyu.edu.tw;

Contributing authors: a979319516@gmail.com;

†These authors contributed equally to this work.

\begin{abstract}
In the traditional electroplating industry of Acrylonitrile Butadiene Styrene (ABS), quality control inspection of the product surface is usually performed with the naked eye. However, these defects on the surface of electroplated products are minor and easily ignored under reflective conditions. If the number of defectiveness and samples is too large, manual inspection will be challenging and time-consuming. We innovatively applied Additive Manufacturing (AM) to design and assemble an automatic optical inspection (AOI) system. The system can identify defects on the reflective surface of the plated product. Based on the deep learning framework from YOLO, we successfully started the neural network model on GPU using the family of YOLO algorithms: from v2 to v5. Finally, our efforts showed an accuracy rate over an average of 70 percentage for detecting real-time video data in production lines. We also compare the classification performance among various YOLO algorithms. Our efforts of visual inspection significantly reduce the labor cost of visual inspection in the electroplating industry.
\end{abstract}


Keywords: Deep Learning, Acrylonitrile Butadiene Styrene (ABS), YOLO,

Automatic Optical Inspection (AOI)

\section{Introduction}

With the rapid development of the industry of water hardware, the metallization of plastics such as ABS plastic is popularly and widely used. ABS has the best electroplating effect and is the most commonly used material because the metal ions plated are not easy to fall off [? ]. However, plastic is usually a poor conductor and must be attached to its conductive membrane first. At present, the processing technology uses chemical plating or electroless plating to adhere a metal film to the surface of the plastic, followed by subsequent copper plating or nickel plating (and chrome plating). Therefore, we should seriously monitor the final quality of the ABS product under the control of the process.

Automated Optical Inspection (AOI) [2] is very common in product quality of inspection applications. Generally speaking, production lines need to use AOI for inspection, mainly for products with large and stable production volumes or products with rigorous product quality inspection. Representatives of such industries include PCB, semiconductor industry, cell phone parts, medical devices, etc. However, with the increasing number of defect detection items, traditional AOI requires the continuous development of new algorithms for different defect characteristics, which is costly and takes a relatively long time to develop. In addition, the algorithm of the traditional program is susceptible to noise interference, and it is less stable against external interference. However, detecting reflective objects, such as electroplated objects, is easy to judge the defects due to the reflection of a metal surface. In contrast, deep learning in artificial intelligence is trained by thousands or even millions of defect photos, which is more flexible and resistant to external interference than traditional AOI. Therefore, we study this interesting issue based on the YOLO framework of electroplated object detection[3-5]. You only look once (YOLO) is a state-of-the-art, real-time object detection system [6], YOLO is developed on a single convolutional network, which simultaneously predicts multiple bounding boxes and class probabilities for those boxes. YOLO trains on full images and directly optimizes detection performance. [7]. This special detection system had revealed its power in many fields; for example, Li et al. [8] used YOLO v3 for the target detection of electronic components on PCB to achieve quality control of accuracy more than 90 percentage. Tian et al. (2019) used YOLO to detect fruits in orchards for judging growth stages of apples. Liu and Wang [9] proposed the YOLO to classify the diseases and pests for tomatoes. In addition, this YOLO is also valuable in mask detection during COVID-19 [10].

We developed an AOI prototype and assembled it with additive manufacturing (AM) technology. The AOI prototype is designed and created by ourselves for detecting the defects on electroplated ABS products, which is 
integrated with Arduino $\mathrm{C}++$ programming to control the motor, inspection light source, stepping motor, robot arm, other related electronic parts, etc. The video captured from the AOI prototype is sent to YOLO models for deep learning to classify products' defects real time. We apply the Python OpenCV image library for image pre-processing, and the processed images are labeled with defect types and locations on each product image. After that, we train the customized YOLO weights by our dataset to classify the defects of products among various YOLO algorithms: from $\mathrm{v} 2$ to $\mathrm{v} 5$ in this study. The detecting performance from different YOLO frameworks is also compared. We organize this study as follows. Section 1 briefly describes the background and motivation of the study; Section 2 explains the hardware and software tools and theoretical methods used. Section 3 describes the process of this study, the description of the self-assembled machine and the calculation results, and Section 4 provides the conclusion and recommendations. In brief, our contributions are summarized as follows: 1 . We assemble a customized AOI assisted by 3D additive manufacturing to detect the defectives of electroplated products (ABS). 2. The algorithm of AOI is launched by the deep learning schema from the YOLO family: from v2 to v5. 3. We compare the performance of various versions of YOLO and discuss their characteristics.

The paper is organized as follow. Section 2 describes the technology background of the overall methodology. Section 3 presents the AOI platform and the YOLO experiments. Section 4 describes the experimental results and prepare the ROC for discussion. The last section is the conclusion including recommendations.

\section{Technology Background}

We prepare the necessary knowledge as follows.

\subsection{Automated Optical Inspection}

Early in the 1990s, the theory of machine vision became mature and entered into industrial applications. At the same time, the field of machine vision has also been in rapid development, and the $\mathrm{m}$ xxxb ost representative of machine vision in industrial applications is automated optical inspection (AOI) [11], it is simply explained as shown in Fig.1.

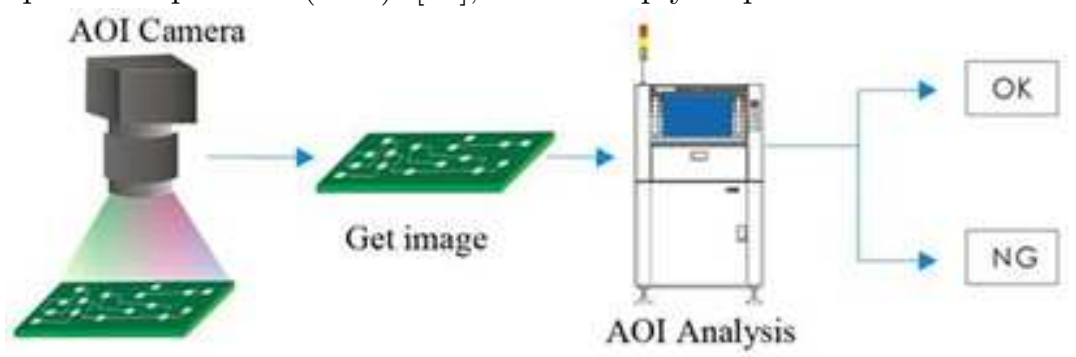

Fig. 1. Operation of AOI 
Source: https://aoi-spi.com/detection-principle-automatic-optical-inspectionaoi/

AOI system early application in textile, packaging, automotive, machinery, etc., the main focus on the speed of detection requirements, the introduction of $\mathrm{AOl}$ inspection system, significantly improve the detection speed [12]. Still, this stage of the AOl inspection system detects larger objects and defects and must compete with the human eye. Hence, the development of this stage of the AOI inspection system simulates the naked eye performance and speed, limited by the time. Although the pace of inspection can meet the requirements, there is no demand for too fine or high precision in the past AOI inspection items, and the development of the resolution of the inspection has not been greatly improved [11].

However, with the industrial development in Taiwan, technology industries such as semiconductors, electronics, biotechnology, and optoelectronics have emerged in the past two decades. Unlike traditional products, technology products are usually more complex, high-precision, tiny, and less tolerant, and any problems in the process may lead to errors in the whole system. In the technology products, the small size and lightweight characteristics, if the AOI optical image inspection, the required resolution is relatively precise so as to push the need of AOI. [13]. The development of AOI has dramatically reduced the error rate of manual visual inspection, thus improving the production control and quality inspection speed, which is what the manufacturing industry expects. The improvement in resolution is also due to the introduction and application of the CCD sensor (Charge-coupled Device, CCD). The image area and pixel resolution to get better data. However, considering CCD in price competition, the AOI system has the advantage of low cost. Therefore, many industries began to introduce AOI systems to replace manual inspection. In most of the technology industry and the demand for inspection of production, the overall booming development of automated optical inspection, creating the growth and demand for the AOI industry. We can find some researches for AOI; for example, since AOI is very sensitive to environment, Chen and Perng [14] proposed a system of a coaxial light, a back light and a motion control to clearly capture the characteristics of statistical textures of the molding surface.

Acciani et al. [15] developed the diagnosis as a pattern recognition problem with a neural network (NN) approach. Five types of solder joints have been classified to perform the diagnosis with a high recognition rate by NN. Richter and Streitferdt [16] proposed a smart AOI paradigm, which uses a superposition of active and unsupervised learning to build a fully annotated dataset while training a suitable classifier by a deep learning cluster analysis. As the development of hardware is fast and the deep learning technology is more popular today, the AOI combined with evolutionary algorithm such as NN will play an import role in the smart sensing network [17]. 


\subsection{YOLO Framework}

In image processing, a kernel, or so called a convolution matrix is a small matrix used for blurring, sharpening, embossing, edge detection, and more. This is accomplished by doing a convolution between a kernel and an image [18-19]. Consider an image has a matrix form given a width $w$ and a height $h$ to store the pixel value in $(x, y)$. Assume $z_{1}, z_{2} \in(x, y)$, then the kernel is defined as a function $\mathrm{k}$ that for all $z \in R^{2}$ satisfies

$$
k\left(z_{1}, z_{2}\right)=<\phi_{1}, \phi_{2}>^{p}
$$

Here $k$ is a matrix, $p$ is a positive integer and $<\phi_{1}, \phi_{2}>^{p}$ is a power form between two vectors: $\phi\left(z_{1}\right)$ and $\phi\left(z_{2}\right)$. For example, if we take $p=2$, then we have

$$
\begin{aligned}
& <\phi_{1}, \phi_{2}>^{2}=\phi\left(z_{2}\right)^{t}\left[\phi\left(z_{1}\right) \phi\left(z_{1}\right)^{t}\right] \phi\left(z_{2}\right) \\
& \phi\left(z_{i}\right) \text { satisfies the following condition: } \\
& \phi: z_{i} \longrightarrow F
\end{aligned}
$$

Where $z$ is the input space and $F$ is the output/feature space. For example, taking the neural network model, the input nodes are $z$, and the final weighed outputs/nodes are $F$. For the two-dimensional data, let $S$ be a given finite set and

$$
S=\left[z_{1}, z_{2}, \ldots, z_{l}\right]
$$

we can propose the $k$ in a matrix form as follows:

$$
K^{p}=\left[\begin{array}{ccccc}
<\phi\left(z_{1}\right), \phi\left(z_{1}\right)>^{p}, & <\phi\left(z_{1}\right), \phi\left(z_{2}\right)>^{p}, \ldots & & <\phi\left(z_{1}\right), \phi\left(z_{l}\right)>^{p} \\
<\phi\left(z_{2}\right), \phi\left(z_{1}\right)>^{p}, & <\phi\left(z_{2}\right), \phi\left(z_{2}\right)>^{p} & \ldots & <\phi\left(z_{2}\right), \phi\left(z_{l}\right)>^{p} \\
\vdots & \vdots & \ddots & \vdots \\
<\phi\left(z_{l}\right), \phi\left(z_{1}\right)>^{p}, & <\phi\left(z_{l}\right), \phi\left(z_{2}\right)>^{p}, \ldots & ,<\phi\left(z_{l}\right), \phi\left(z_{l}\right)>^{p}
\end{array}\right]
$$

Here Convolution $K^{p}$ is a general purpose filter effect for images. Simply speaking, we can extract the information from image by setting the filter and stride of kernel, and the padding of feature. Therefore, a basic framework of CNN is shown as follows [20]:

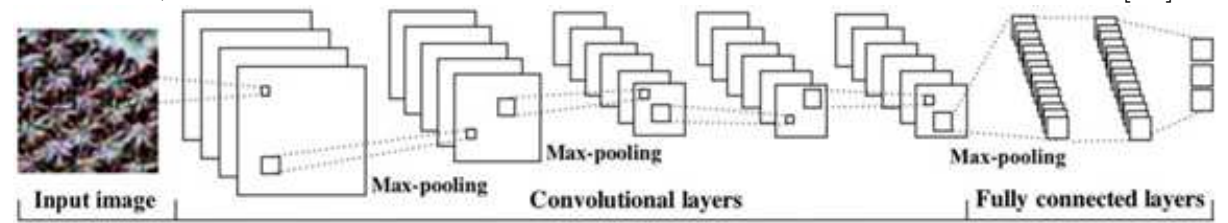

Fig. 2. CNN Framework

Source: [20]

This CNN structure was first proposed by Fukushima [21]. The development of CNNs is to solve the tough challenge of image recognition and detection. A CNN consists of an input layer, we may recall the input vector 
$\mathrm{z}$ above: a vector of pixels to represent an image; moreover, one or more hidden layers, and an output layer (Fig. 2). These mathematical layers help the computer to define the details of the image bit by bit, in order to eventually distinguish a specific object. The convolution layers are designed to be cyclic for reducing the scale of image, and these convolutions are used to filter the input data and find information. The convolutional layers perform most of the heavy computational work in a CNN, acting as mathematical filters to help the computer find image edges, dark and light areas, colors, and other details such as height, width, and depth. And the pooling layer is usually sandwiched between layers to reduce the size of the representation created by a volume layer, and to reduce the need for memory to accommodate more layers. Finally, the fully connected layer is used to connect neurons in one layer to all neurons in another layer. The outputs will be arranged to various classification after the fully connected layers.

YOLO is an abbreviation of "You Only Look Once". YOLO algorithm uses neural networks to provide real-time object detection. The critical components of YOLO algorithms are CNNs [22]. The basic framework of YOLO is shown in Fig. 3 [6].

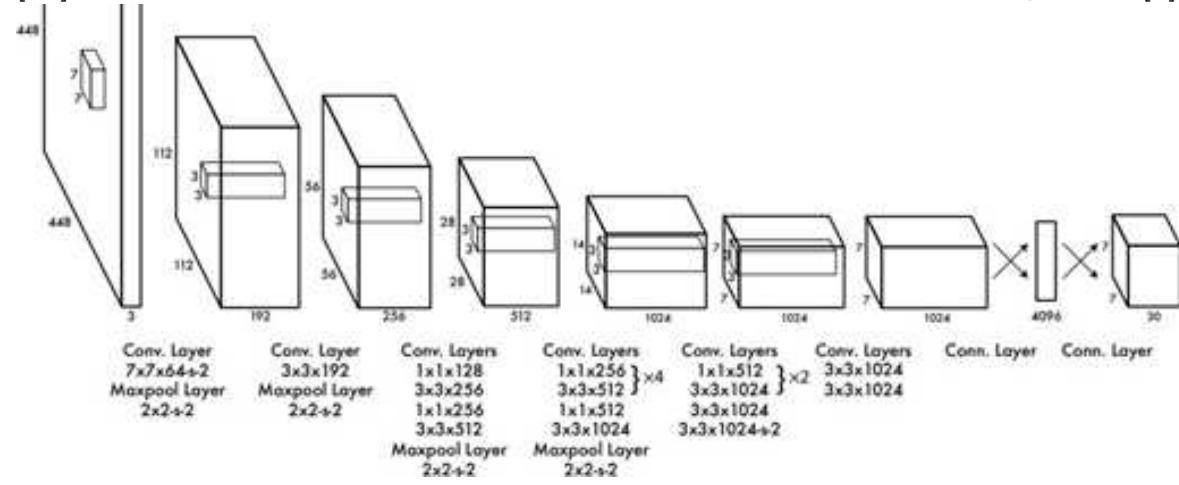

Fig. 3. YOLO Framework

Source: [6]

YOLO can determine the class and location of the object in the image with only one CNN, which significantly improves the recognition speed. The whole network design is point-to-point, easy to train, and fast. The critical difference between Convolutional Neural Network (CNN) [23] and traditional neural networks [24] is that the human retina inspires CNNs' visual system. CNN models are validated to perform excellently in image processing, such as LetNet [24], the progenitor of CNNs, and AlexNet [25], which started to make a name for itself in the field of machine vision. In 1998, Yann LeCun et al. published "LeNet" as the original CNN framework, which formally brought the concept of convolution into machine learning. The LeNet network architecture uses MaxPooling to strengthen the features, and the output layer uses Radial Basis Function radially oriented Euclidean distance function. The distance function is used in the output layer. 
YOLO requires a neural network framework for training, and for this we have used DarkNet (https://pjreddie.com/darknet/yolo/). The first version of the input image of $448 \times 448$, which has 26 layers, with 24 Convolution Layers followed by 2 fully connected layers. The major problem with The first YOLO: YOLOv1 is limited to its inability to detect microscopic objects. After the first version, many researchers and scholars try to extend the source code for better performance. After the first version, many researchers and scholars try to extend the source code for better performance. For example, YOLOv2 includes batch normalization layers after each convolutional layer and has thirty layers compared to YOLOv1 of twenty-six layers. Moreover, this version introduced the concept of anchor boxes. Anchor boxes are predefined boxes provided by the user to Darknet, which gives the network an idea about the relative position and dimensions of the objects to be detected. It has to be calculated using the training set objects. YOLOv3 evolves to have one hundred and six layers of neural network. And it can Detection on three scales for detecting objects of small to considerable size. This version provides nine anchor boxes: three per scale. Most important, the multiclass problem turned into a multilabel problem. And YOLOv3 is powerful in detecting tiny objects. Yolov4 was recently released in 2020, and two Taiwanese scholars from Academia Sinica joined this project [26]. According to the work of Bochkovskiy et al., YOLOv4 is optimal for real-time object detection tasks because its performance lies on the Pareto optimality curve of the accuracy and speed of frames per second (FPS). YOLOv5 is funded by the open source by Ultralytics (https://ultralytics.com/)[27]. Ultralytics was founded in 2014, spearheading several U.S. Intelligence Community (IC) and Department of Defense (DoD) initiatives in the fields of particle physics, data science and artificial intelligence.

We review the papers integrating YOLO algorithms and AOI for the quality detection of electroplating industry; however, the paper number of this field is quite less. Most of these contribution focus on the initiative of printed circuit board (PCB). Therefore, this study should be valuable for intelligent manufacturing of small and medium enterprises (SMEs). Primarily we focus on the tiny defects on the ABS product by applying various YOLO algorithms: from v2 to v5.

\section{Methodology}

First, we design the AOI prototype for the customized needs of the study. Second, we use the AOT prototype to capture the necessary videos and divide them into FPS to label the defective images. Finally, we apply different YOLO algorithms to compare their performance for the same validation set of images. We designed the AOI prototype by CAD and assembled it with parts from additive manufacturing technology (3D printing) for the customized joints, which is shown in Fig. 4 (from left to right: CAD file, prototype and conveyor).

\footnotetext{
${ }^{0}$ text of the footnote
} 

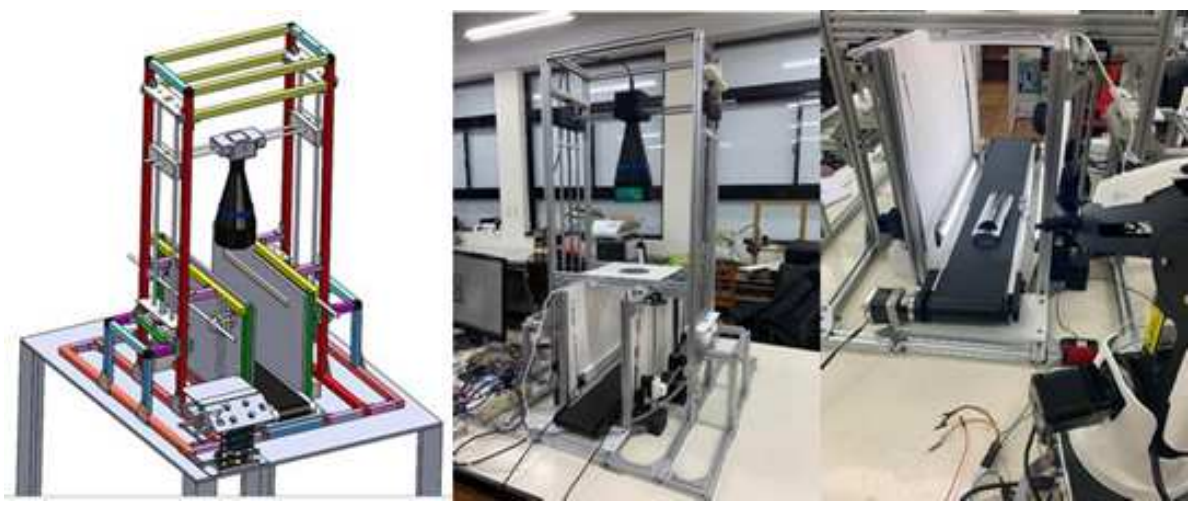

Fig. 4. CAD and AOI Prototype

We use the series of Arduino boards to connect the stepping motor, light source (LED), Dobot robot arm (https://www.dobot.cc/), conveyor, and ultrasonic sensor to finish the AOT prototype. We designed the prototype by CAD and assembled it with parts from additive manufacturing technology (3D printing) for the customized joints. We found the image quality is very sensitive to our lab environment. In this study, we try to capture the curved shape of the detected object; the defect characteristics of the curved shape will be challenges if a CCTV lens is selected. Therefore, this study uses a telecentric lens [28] with parallel lights (LED) to set up the AOI device to capture the defect images by flattening this non-planar feature. Telecentric lens is designed in response to image size variation or image distortion and deformation caused by the different working distances of Closed-circuit television camera (CCTV) lenses. The comparison of Telecentric lens and CCTV lenses is shown in Fig. 5 (Left: CCTV, Right: Telecentric).
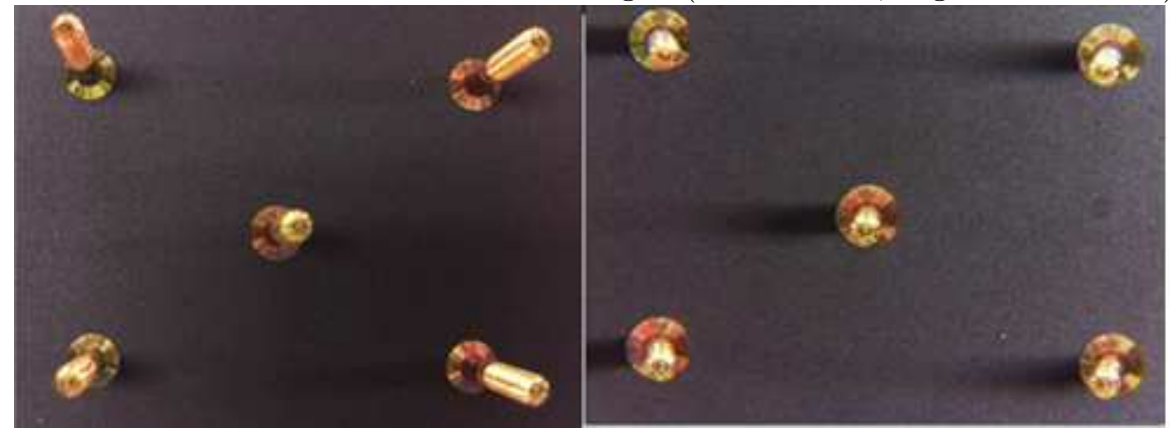

Fig. 5. Comparison of Telecentric Lens (Right) and CCTV Lens(Left)

The characteristic of a telecentric lens is that it can obtain the same size image for the same size object to be measured at different working distances and is generally used for dimensional measurement or surface inspection on curved and curved products. is designed in response to image size variation or image distortion and deformation caused by the different working distances 
of CCTV lenses. The characteristic of a telecentric lens is that it can obtain the same size image for the same size object to be measured at different working distances and is generally used for dimensional measurement or surface inspection on curved and curved products.

Therefore, after several try-and error experiments, we use a pair of bar LED with the trigger of the arrival of product waiting for detection. In machine vision, the light source accounts for a lot of weight in the inspection process. The critical point of the lighting design is to highlight the essential features for detection, suppress the unimportant background. To obtain a good image, we must understand the object's surface characteristics to be tested through try and error experiments to select the appropriate light source to facilitate the lens to capture the defective features of the tested object. if the brightness of the light source is too high or too low, the choice of light source If the brightness of the light source is too high or too low, the type of light source is not suitable, the wrong way of lighting will significantly affect the final image of the camera. This special capture of product image is shown in Fig. 6 by setting a pair of bar LED.

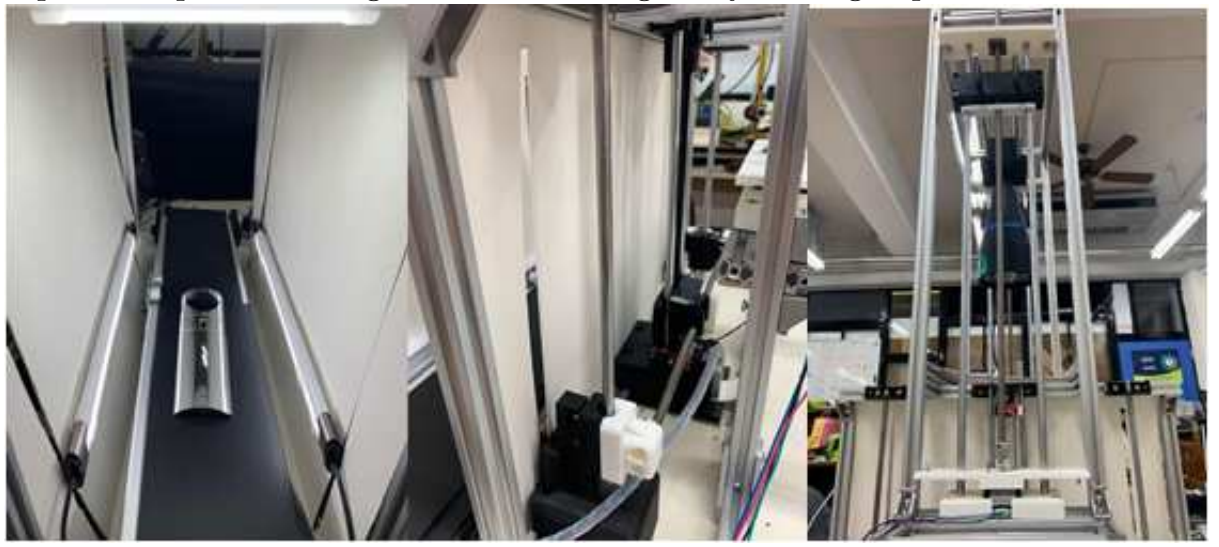

Fig. 6. LED Mechanism for Capturing the Product Image

The tested products are fed into this AOI machine one by one by the conveyor. The stepping motors are used to move up and down to complete the light source's illumination at different angles; therefore, this interesting AOI machine can reveal the defects of the global surface of the product. At the same time, OpenCV combined with the camera is used to take images and recognition. We design this AOI machine that the conveyor will transmit the ABS product one by one, and the camera will record the video during operation. Once the video is captured, the robot arm will remove the undesirable product at the end of the conveyor. The defective situation makes the removal decision of the ABS product. All YOLO algorithms form v2 to v4 (https://github.com/AlexeyAB) are developed and implemented on the Nvidia GPU server based on Linux of the center of artificial intelligence (AI) at Da-Yeh University. And the YOLO v5 is provided by Ultralytics 
(https://ultralytics.com). The preparation of image processing is introduced as follows: first, we need to label the defective products by reducing the image scale to increase the recognition efficiency. This study uses the LabelImg (https://github.com/tzutalin/labelImg) as an image marker tool as a dataset for the deep learning model of defect images. LabelImg is an image marker tool with open-source code. This type of file can record the defect coordinates marked on the image and provide a deep learning model for training.Second, we set HSV (Hue, Saturation, Value) mask to filter the objects other than the plated objects to reduce the image noise by using ConvertScaleAbs function in OpenCV (https://opencv.org/) to enhance the contrast. The contrast enhancement is done by using the ConvertScaleAbs function, as shown in Fig. 6 (left). Finally, the enhanced images are sharpened by using Filter2D convolutional algorithm, which is the pre-training labeling process of images, as shown in Fig. 6 (right). This study collected 508 defective images of surface impurities from electroplated parts. The ratios of training, testing and validation of these images are percentages of 60, 30 and 10, respectively. We labeled the data as two categories: the defective category, including speck and pock, and the non-defective category, to build the training, testing, and validating set. Once the data above is ready, we apply four YOLO algorithms: v2, v3, $\mathrm{v} 4$, and $\mathrm{v} 5$ to validate the discrimination power among various algorithms.

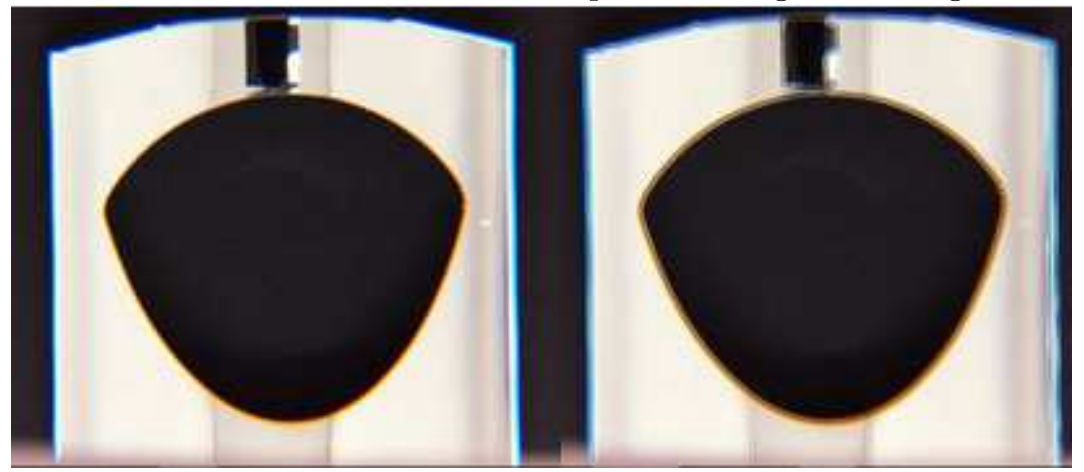

Fig.7. Preprocessing of Images: Enhanced (Left) and Sharpened (Right) with Different Kernels

\section{Experimental Results}

We labeled 508 images for this study, and the input size of image is $416 \mathrm{x}$ 416. The model parameters of YOLO algorithms are summarized as in Table 1 , the corresponding parameters are customized to prevent breakdown/nonconvergence during training. The iteration number is enlarged accordingly because YOLOv4 and YOLOv5 have more complicated framework than YOLOv3 and YOLOv2. Decision-makers should decide these parameters to meet their customized needs; this could be considered case by case. 
Table 1 Model Parameters

\begin{tabular}{lllll}
\hline Model Parameters & YOLOv2 & YOLOv3 & YOLOv4 & YOLOv5 \\
\hline Batch & 64 & 64 & 64 & 64 \\
Max Batches & 5000 & 5000 & 5000 & 1000 \\
Learning Rate & $10^{-5}$ & $10^{-4}$ & $10^{-4}$ & $10^{-4}$ \\
\hline
\end{tabular}

The paprameters are set according to the model scale.

When we compare the behavior characteristics of these YOLO models; for example, v2, v3 and v4. The loss values reduce more steeper and faster from v2 to v4 (see Fig. 8) during iterations. This means the model evolves more effectively from $\mathrm{v} 2$ to $\mathrm{v} 4$. The average losses of $\mathrm{v} 2$, v3 and $\mathrm{v} 4$ are $5.79,4.91$ and 2.73 , respectively. The training performance of average loss also shows that the $\mathrm{v} 4$ is superior to $\mathrm{v} 3$, and the v3 is superior to v2. We also check the performance of YOLO v5, which is controversial when compared with YOLO v5 by its originality. However, according to our experiment in this study, In terms of loss, YOLO v5 is better, but the discrimination power of two models are almost the same, we will present the results later. The YOLO v5 is much smaller, which is very suitable for Edge AI users. The evolution of loss of YOLO v5 is shown in Fig. 9.

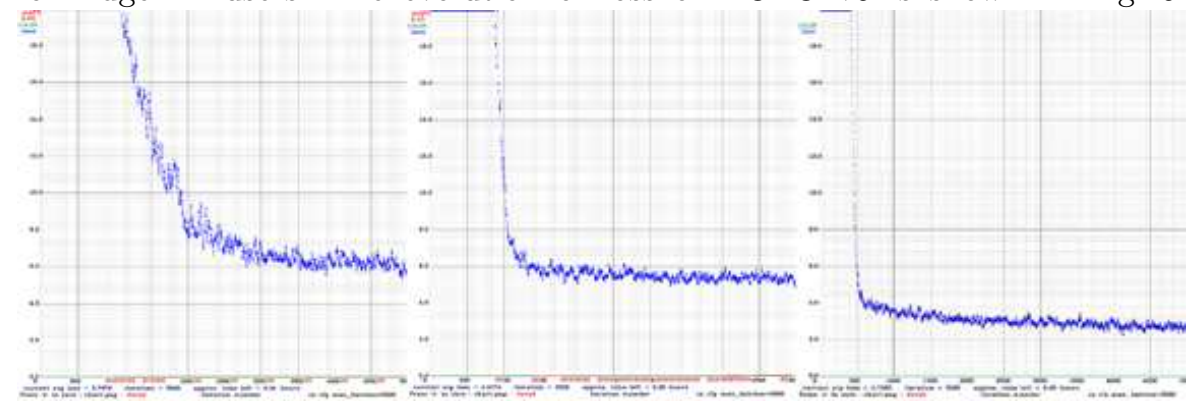

Fig.8.Loss Evolution with Iterations (Left: v2, Middle: v3 and Right: v4) 


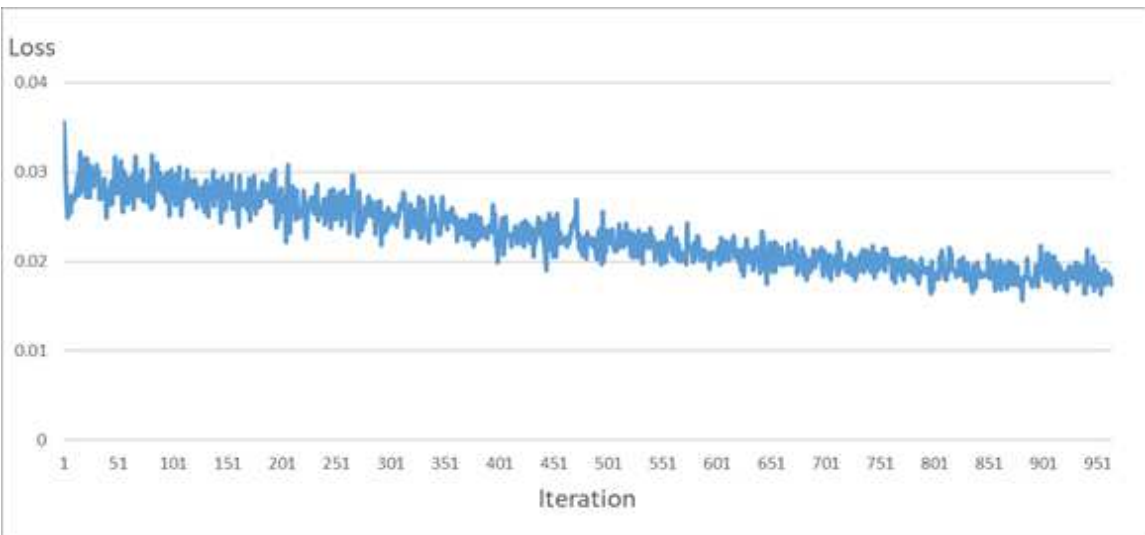

Fig.9. Loss Evolution with Iterations by YOLO v5

An ROC curve (receiver operating characteristic curve) is an effective tool to compare the performance of the YOLO-classification models of this study. The confusion matrix is the basis of ROC curve, and it is also the most basic, intuitive, and simple method to measure the accuracy of classification models [29]. The predictive performance of the categorical models is statistically presented in four categories by confusion matrix, which is shown in Fig. 10.

\begin{tabular}{|c|c|c|}
\hline Predict. Actual & $\begin{array}{l}\text { Actually Defective. } \\
\text { (Positive) }\end{array}$ & $\begin{array}{l}\text { Actually Non-defective. } \\
\text { (Negative) }\end{array}$ \\
\hline $\begin{array}{l}\text { Predicted Defective . } \\
\text { (Positive) }\end{array}$ & $\begin{array}{l}\text { Predicted as defective. } \\
\text { Actually is defective. } \\
\text { (True Positive TP) }\end{array}$ & $\begin{array}{l}\text { Predicted as defective. } \\
\text { Actually is non-defective. } \\
\text { (False Positive: FP). }\end{array}$ \\
\hline $\begin{array}{l}\text { Predicted Non-defective } \\
\text { (Negative) }\end{array}$ & $\begin{array}{l}\text { Predicted as non-defective. } \\
\text { Actually is defective } \\
\text { (False Negative: FN) }\end{array}$ & $\begin{array}{l}\text { Predicted as non-defective- } \\
\text { Actually is non-defective: } \\
\text { (True Negative: TN). }\end{array}$ \\
\hline
\end{tabular}

Fig. 10. Confusion Matrix

We continue to evaluate the model's performance by calculating Accuracy, Precision, Recall, and F1-Score through four categories of statistics and use Recall as the primary reference for identifying the version among YOLO models. Here are the mathematical definitions for Accuracy, Precision, Recall, and $F 1-S c o r e$ : 


$$
\begin{aligned}
& \text { Accuracy }=\frac{T N+T P}{T N+T P+F N+F P} \\
& \text { Precision }=\frac{T P}{T P+F P} \\
& \text { Recall }=\frac{T P}{T P+F N} \\
& F 1-\text { Score }=\frac{2 * \text { Precision } * \text { Recall }}{\text { Precision }+ \text { Recall }}
\end{aligned}
$$

Moreover, the evaluation results of various models are summarized in Table 2. Interestingly, the Yolov3 and Yolov5 performed in a balanced scope in Accuracy, Precision, Recall, and F1-Score, simultaneously. And the accuracy of YOLOv2 is somewhat lower than we expected. However, we found overfitting and overemphasized phenomenon for the detected results from YOLOv4 and YOLOv5 (see Fig. 11). When we compare these detected pictures from v3, v4, and v5 altogether, as in Fig. 11, we can easily find too many (fake) points of speck and pock generated by $\mathrm{v} 4$, and we think this causes the low performance of YOLOv4. YOLOv5 also has the same problem. We guess this issue resulting from the YOLOv4 and YOLOv5 have larger and complicated CNNs; however, the data of this study is limited. Therefore, if we didn't provide adequate data for them, then they won't perform best as we expect. The overfitting effect is more severe for YOLOv4 than YOLOv5.

Table 2 Model Evaluation

\begin{tabular}{lllll}
\hline Model Performance & YOLOv2 & YOLOv3 & YOLOv4 & YOLOv5 \\
\hline TP & 0.18 & 0.71 & 0.71 & 0.75 \\
FP & 0.00 & 0.17 & 0.31 & 0.24 \\
FN & 0.82 & 0.29 & 0.29 & 0.25 \\
TN & 1.00 & 0.83 & 0.69 & 0.76 \\
Accuracy & 59 & 71 & 70 & 75 \\
Precision & 100 & 81 & 69 & 76 \\
Recall & 18 & 77 & 69 & 75 \\
F1-Score & 30 & 77 & 69 & 75 \\
\hline
\end{tabular}

Here $\mathrm{TP}+\mathrm{FN}=1$ and $\mathrm{FP}+\mathrm{TN}=1$

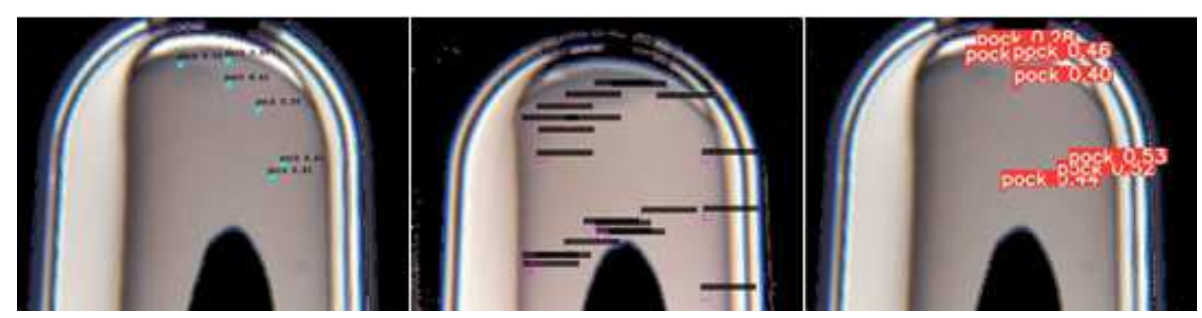

Fig. 11. Detected Results from Various YOLO Algorithms (Left: v3, Middle: 
v4, Right: v5)

\section{Conclusions and Recommendations}

In this study, we successfully solve the quality detection problem of the electroplating industry by combining the YOLO technology and the customized prototype of the AOI inspection platform. After the actual validation, YOLOv3 and YOLOv5 perform in a balanced scope with an accuracy rate of over 70 pervent. Although YOLOv2 performs poorly, we found that YOLOv2 is more accurate in the large area of pock detection (large object). A decision-maker can arbitrarily assign weights for multiple criteria of Accuracy, Precision, Recall, and F1-Score, to select the final model to launch in practice.

The study results validate that YOLOv3 and YOLOv5 have the advantage of overall other models combined with the AOI system for better quality detection. YOLOv4 will show its value if we can provide enough data. Shortly, how to set the model parameters properly is a primary task worthy of exploration. In addition, more defective images should be automatically collected to reduce the overfitting/overemphasized phenomena for YOLOv4 and YOLOv5.

Finally, we recommend increasing the number of pieces for each defect model and controlling the difference in the number of samples for each defect to provide the model with valuable training data.

\section{References}

[1] Teixeira, L.A.C., and Santini, M.C. (2005). Surface conditioning of ABS for metallization without the use of chromium baths. Journal of Materials Processing Technology, 170, 37-41. https://doi.org/10.1016/j.jmatprotec.2005.04.075. [2] Tsai, Y. H., Lyu, N. Y., Jung, S. Y., Chang, K. H., Chang, J. Y., and Sun, C. T. (2019). Deep Learning Based AOI System with Equivalent Convolutional Layers Transformed from Fully Connected Layers. In 2019 IEEE/ASME International Conference on Advanced Intelligent Mechatronics (pp. 103-107). IEEE. https://doi.org/ 10.1109/AIM.2019.8868602.

[3] Tian, Y., Yang, G., Wang, Z., Wang, H., Li, E., andLiang, Z. (2019). Apple detection during different growth stages in orchards using the improved YOLOV3 model. Computers and Electronics in Agriculture, 157(January), 417-426. https://doi.org/10.1016/j.compag.2019.01.012.

[4] Xie, Y., Cai, J., Bhojwani, R., Shekhar, S., and Knight, J. (2020). A locally-constrained yolo framework for detecting small and densely-distributed building footprints. International Journal of Geographical Information Science, 34(4), 777-801. https://doi.org/10.1080/13658816.2019.1624761.

[5] Liu, E., Chen, K., Xiang, Z., and Zhang, J. (2020). Conductive particle detection via deep learning for ACF bonding in TFT-LCD manufacturing. Journal of Intelligent Manufacturing, 31(4), 1037-1049. https://doi.org/:10.1007/s10845-019-01494-9. 
[6] Redmon, J., Divvala, S., Girshick, R., and Farhadi, A. (2016). You only look once: Unified, real-time object detection. In Proceedings of the IEEE conference on computer vision and pattern recognition (pp. 779-788). https://doi.org/10.1109/CVPR.2016.91.

[7] Plastiras, G., Kyrkou, C., andTheocharides, T. (2018). Efficient convnet-based object detection for unmanned aerial vehicles by selective tile processing. ACM International Conference Proceeding Series. https://doi.org/10.1145/3243394.3243692.

[8] Li, J., Gu, J., Huang, Z., andWen, J. (2019). Application research of improved YOLO V3 algorithm in PCB electronic component detection. Applied Sciences (Switzerland), 9(18). https://doi.org/10.3390/app9183750. [9] Liu, J., andWang, X. (2020). Tomato Diseases and Pests Detection Based on Improved Yolo V3 Convolutional Neural Network. Frontiers in Plant Science, 11(June), 1-12. https://doi.org/10.3389/fpls.2020.00898.

[10] Loey, M., Manogaran, G., Taha, M. H. N., andKhalifa, N. E. M. (2021). Fighting against COVID-19: A novel deep learning model based on YOLOv2 with ResNet-50 for medical face mask detection. Sustainable Cities and Society, 65(October 2020), 102600. https://doi.org/10.1016/j.scs.2020.102600. [11] Lu, R. S., Shi, Y. Q., Li, Q., and Yu, Q. P. (2010). AOI techniques for surface defect inspection. In Applied mechanics and Materials (Vol. 36, pp. 297-302). Trans Tech Publications Ltd. https://doi.org/10.4028/www.scientific.net/AMM.36.297.

[12] Perng, D. B., Chen, Y. C., and Lee, M. K. (2005). A novel AOI system for OLED panel inspection. In Journal of Physics: Conference Series (Vol. 13, No. 1, p. 081). IOP Publishing. https://doi.org/10.1088/1742-6596/13/1/081 [13] Fan, K. C., and Hsu, C. (2005). Strategic planning of developing automatic optical inspection (AOI) technologies in Taiwan. In Journal of Physics: Conference Series (Vol. 13, No. 1, p. 090). IOP Publishing. https://doi.org/10.1088/1742-6596/13/1/090.

[14] Chen, S. H., and Perng, D. B. (2016). Automatic optical inspection system for IC molding surface. Journal of Intelligent Manufacturing, 27(5), 915-926. https://doi.org/10.1007/s10845-014-0924-5.

[15] Acciani, G., Brunetti, G., and Fornarelli, G. (2006). Application of neural networks in optical inspection and classification of solder joints in surface mount technology. IEEE Transactions on industrial informatics, 2(3), 200-209. https://doi.org/10.1109/TII.2006.877265

[16] Richter, J., and Streitferdt, D. (2019). Modern architecture for deep learning-based automatic optical inspection. In 2019 IEEE 43rd Annual Computer Software and Applications Conference (COMPSAC) (Vol. 2, pp. 141-145). IEEE. https://doi.org/10.1109/COMPSAC.2019.10197.

[17] Liu, G., Nouaze, J. C., Touko Mbouembe, P. L., and Kim, J. H. (2020). YOLO-tomato: A robust algorithm for tomato detection based on YOLOv3. Sensors, 20(7), 2145. https://doi.org/10.3390/s20072145.

[18] Shawe-Taylor, J., and Cristianini, N. (2004). Kernel methods for pattern analysis. Cambridge university press. 
[19] Takeda, H., Farsiu, S., and Milanfar, P. (2007). Kernel regression for image processing and reconstruction. IEEE Transactions on image processing, 16(2), 349-366.

[20] Li, W., Dong, R., Fu, H., and Yu, L. (2019). Large-scale oil palm tree detection from high-resolution satellite images using two-stage convolutional neural networks. Remote Sensing, 11(1), 11. https://doi.org/10.3390/rs11010011.

[21] K. Fukushima, K. (1989). A Hierarchical Neural Network Capable of Visual Pattern Recognition. Neural Network. Vol. 1, 1989. https://doi.org/10.1016/0893-6080(88)90014-7

[22] Albawi, S., Mohammed, T. A., and Al-Zawi, S. (2017). Understanding of a convolutional neural network. In 2017 International Conference on Engineering and Technology (ICET) (pp. 1-6). IEEE.10.1109/ICEngTechnol. https://doi.org/2017.8308186.

[23] LeCun, Y., and Bengio, Y. (1995). Convolutional networks for images, speech, and time series. The handbook of brain theory and neural networks, 3361(10), 1995. https://doi.org/10.3902/jnns.2.1.

[24] LeCun, Y., Bottou, L., Bengio, Y., and Haffner, P., 1998, Gradient-based learning applied to document recognition. Proceedings of the IEEE, 86(11), 2278-2324. https://doi.org/10.1109/5.726791

[25] Krizhevsky, A., Sutskever, I., and Hinton, G. E., 2012, Imagenet classification with deep convolutional neural networks. In Advances in neural information processing systems, 1097-1105. https://doi.org/10.1016/j.aasri.2014.05.013

[26] Bochkovskiy, A., Wang, C. Y., and Liao, H. Y. M. (2020). Yolov4: Optimal speed and accuracy of object detection. arXiv preprint arXiv:2004.10934. https://doi.org/10.1093/qjmed/hcu035

[27] Ting, L., Baijun, Z., Yongsheng, Z., and Shun, Y. (2021). Ship Detection Algorithm based on Improved YOLO V5. In 2021 6th International Conference on Automation, Control and Robotics Engineering (CACRE) (pp. 483-487). IEEE. https://doi.org/10.1109/CACRE52464.2021.9501331.

[28] Li, D., and Tian, J. (2013). An accurate calibration method for a camera with telecentric lenses. Optics and Lasers in Engineering, 51(5), 538-541. https://doi.org/10.1016/j.optlaseng.2012.12.008.

[29] Bradley, A. P. (1997). The use of the area under the ROC curve in the evaluation of machine learning algorithms. Pattern recognition, 30(7), 11451159. https://doi.org/10.1016/S0031-3203(96)00142-2

\section{Declarations}

- Funding

This research is an independent research from my lab H560 in Da-Yeh University (no official funding).

- Conflict of interest/Competing interests (check journal-specific guidelines for which heading to use)

We declare that we have no known competing financial interests or personal 
relationships that could have appeared to influence the work reported in this paper.

- Ethics approval We declare this research is original and innovative.

- Consent to participate We delared that we contribute equally to this work

- Consent for publication We agree with the Copyright Transfer Statement later.

- Availability of data and materials Yes (after acceptance)

- Code availability Yes (after acceptance)

- Authors' contributions We (two authors) contributed equally to this work. 


\section{Supplementary Files}

This is a list of supplementary files associated with this preprint. Click to download.

- defectFPStestsample.avi

- waterwarevideorealtimetest.mp4 